Check for updates

Cite this: RSC Adv., 2018, 8, 26755

Received 4th May 2018

Accepted 12th July 2018

DOI: $10.1039 / \mathrm{c} 8 \mathrm{ra0} 8846 \mathrm{e}$

rsc.li/rsc-advances

\title{
Development and characterisation of self- assembled graphene hydrogel-based anodes for bioelectrochemical systems
}

\author{
Mariela I. Lescano, ${ }^{a}$ Aurelien Gasnier, (D) ${ }^{\mathrm{b}}$ Maria L. Pedano, ${ }^{\text {cd }}$ Mauricio P. Sica, ${ }^{\text {ad }}$ \\ Daniel M. Pasquevich ${ }^{a}$ and Maria B. Prados (D) *ad
}

\begin{abstract}
In this work, we report a simple and scalable method to produce high efficiency 3D graphene-based electrodes $(\mathrm{GH})$ for bioelectrochemical systems. GH were obtained by self-assembly of graphene oxide, through slow reduction with ascorbic acid over conductive mesh-works (carbon cloth and stainlesssteel). The GH structure and composition were characterised by electron microscopy (SEM) and spectroscopy (FTIR and Raman), whereas the electrodes' performance was tested by chronoamperometry and cyclic voltammetry in a microbial electrolysis cell (MEC) inoculated with a pure culture of $G$. sulfurreducens. The hydrogel had a broad pore size distribution $(>1 \mu \mathrm{m})$, which allowed bacterial colonisation within the framework. The macro-porous structure and chemical properties of the hydrogel rendered a higher bacterial loading capacity and substrate oxidation rate than other carbonaceous materials, including different reported graphene electrodes, which significantly increased MEC performance.
\end{abstract}

\section{Introduction}

Bioelectrochemical systems (BES) permit the extraction of energy from microbial anaerobic respiration. These systems are based on electrogenic microorganisms, such as bacteria of the genus Geobacter, that are capable of transferring electrons extra-cellularly during respiration. ${ }^{\mathbf{1 , 2}}$ Basically, these bacteria oxidise organic compounds, such as acetate, in the cytosol and obtain energy catalysing the transference of the electrons through a respiratory chain to a solid final acceptor outside the cell, such as Fe(III) oxides. This final step is accomplished through extra-cellular electron transfer mechanisms (EET), which include direct-contact between the bacterial cell and the electron acceptor through outer-membrane proteins (cytochromes), diffusion of soluble electron shuttles (e.g. flavins, quinones, phenazines) and electron transport through the extra-cellular biofilm matrix (organic compounds synthesised

${ }^{a}$ Instituto de Energia y Desarrollo Sustentable, Centro Atomico Bariloche, Comision Nacional de Energia Atomica, Av. E. Bustillo 9500, 8400 S. C. de Bariloche, Rio Negro, Argentina.E-mail: mbprados@cab.cnea.gov.ar; Tel: +54 2944445107

${ }^{b}$ Gerencia de Investigacion Aplicada, Centro Atomico Bariloche, Comision Nacional de Energia Atomica, CONICET, Av. E. Bustillo 9500, 8400 S. C. de Bariloche, Rio Negro, Argentina

${ }^{c}$ Lab. de Fotonica y Optoelectronica, Centro Atomico Bariloche, Comision Nacional de Energia Atomica, CONICET, Av. E. Bustillo 9500, 8400 S. C. de Bariloche, Rio Negro, Argentina

${ }^{d}$ Instituto Balseiro, Universidad Nacional de Cuyo, Centro Atomico Bariloche, Av. E. Bustillo 9500, 8400 S. C. de Bariloche, Rio Negro, Argentina by the microorganisms). These EET have been described in detail and extensively reviewed..$^{3,4}$

Notably, it has been demonstrated that electrogenic bacteria can use these EET to transfer electrons to an electrode. ${ }^{5}$ The current thus generated can produce useful work in a microbial fuel cell (MFC) ${ }^{6}$ or can be used for the production of valuable products in the cathode. For example, microbial electrolysis cells (MEC) are bio-catalysed electrolysis reactors employed in the production of hydrogen. ${ }^{7}$ Currently, BES represent a promising sustainable green technology to turn organic pollutants present in wastewater into a renewable source of energy. ${ }^{\mathbf{8 - 1 0}}$

Additionally, a new generation of BES emerged, applied to desalination, ${ }^{\mathbf{1 1}}$ electrosynthesis ${ }^{\mathbf{1 2}}$ and remediation of complexly contaminated sediments. ${ }^{\mathbf{1 3 , 1 4}}$ Interestingly, there is growing evidence of the efficiency of BES in the treatment of refractory pollutants, such as pyridine (commonly found in several industrial wastewaters), ${ }^{15}$ chloramphenicol $^{16}$ and azo dye. ${ }^{17}$

However, the development of BES technology has been hampered by different factors, such as a low rate of charge transfer to the electrodes.

In most BES, like MFC and MEC, the anode potential influence the cellular respiration similarly to the concentration of a soluble electron acceptor. Therefore, respiration will occur only at a certain potential, where the anode accepts electrons from the microorganisms and determines their respiration rate. ${ }^{18}$ In order to achieve a high energy output with minimal potential losses, electrogenic microorganisms should produce a high current density at a low anode potential (the closest possible to the redox potential of the substrate 
being oxidised). Improving BES performance requires the structural and material optimisation of the electrode. The optimised structure should increase the active surface area available for bacterial colonisation, while the electrode composition should improve the bacteria-electrode affinity interactions and the catalysis of the electron transfer process.

Recently, graphene has been proposed as a suitable material for electrode applications in $\mathrm{MFC} .{ }^{19}$ Graphene is a singleatom-thick two-dimensional (2D) carbon nano-structured material, where the $\mathrm{sp}^{2}$ hybridisation of all bonds across the sheet gives rise to interesting and unique properties, such as high mechanical strength, flexibility, thermal and electrical conductivity, large specific surface area and stability. ${ }^{\mathbf{2 0 , 2 1}}$ Currently, there are many methods to prepare graphene, such as chemical vapour deposition (CVD), liquid phase exfoliation, mechanical exfoliation, molecular assembly and epitaxial growth. ${ }^{22}$ The graphene product obtained by each of these methods has different qualities, differing in dimension, shape, type and amount of defects, which strongly affect the properties of the material. In addition, the scale of graphene production also depends on the method of synthesis employed. For the preparation of graphene based electrodes for MFC application, different strategies have been applied. Thin-films of graphene sheets used as anodes of MFC, operated with different bacterial species, demonstrated high biocompatibility and a good power performance. ${ }^{23,24}$ However, 2D structures have limited bacterial loading capacity. In addition, the stacking between individual sheets into multilayered graphene, caused by van der Waals attractions from the basal planes, largely reduces the intrinsic specific area, the conductivity and the mechanical strength of individual graphene sheets.

An effective approach to overcome these problems is the synthesis of three-dimensional (3D) graphene architectures, such as hydrogels, foams and sponges. ${ }^{25}$ Yong et al. ${ }^{26}$ have synthesised macro-porous free-standing graphene foam by CVD, employing a nickel foam as substrate, to build anodes for MFC. This architecture substantially increases the electrode surface. However, due to the scarcity of polar organic groups on the surface of the high-quality graphene obtained by CVD, this electrode did not provide anchoring points for bacteria attachment and therefore, it had to be functionalized to provide a significant bacterial loading capacity.

In this work, we present a simple and scalable wet method to prepare graphene hydrogel $(\mathrm{GH})$ based electrodes for BES applications. The assembly of the electrodes involved the chemical preparation of graphene oxide flakes (GO) according to the Tour's method, ${ }^{27}$ followed by a slow reduction with ascorbic acid over different conductive mesh-works, such as carbon cloth (GH-CC) and stainless-steel (GH-SS). These electrodes were tested in single chamber MECs based on Geobacter sulfurreducens, a model electrogenic bacteria. With no functionalization step, our three-dimensional macro-porous design promoted a high BES performance due to bacterial colonisation of the whole electrode framework and a faster substrate oxidation rate.

\section{Materials and methods}

\subsection{Media and bacteria growth conditions}

All the bioelectrochemical assays were performed using Geobacter sulfurreducens. G. sulfurreducens growth medium contained (per litre): $0.1 \mathrm{~g}$ of $\mathrm{KCl}, 1.5 \mathrm{~g}$ of $\mathrm{NH}_{4} \mathrm{Cl}, 0.6 \mathrm{~g}$ of $\mathrm{NaH}_{2} \mathrm{PO}_{4}$, $2.5 \mathrm{~g}$ of $\mathrm{NaHCO}_{3}, 10 \mathrm{ml}$ of vitamin mix and $10 \mathrm{ml}$ of trace mineral mix (Sigma-Aldrich). ${ }^{28}$ The medium was adjusted to $\mathrm{pH}$ 6.8 , sterilised and flushed with $\mathrm{N}_{2}: \mathrm{CO}_{2}(80: 20)$ to remove oxygen. Sodium acetate $(30 \mathrm{mM})$ served as the electron donor and as the carbon source and sodium fumarate as the electron acceptor $(40 \mathrm{mM})$. Cells were cultured at $30{ }^{\circ} \mathrm{C}$. Steady-state cells were anaerobically transferred to the electrochemical cell $(10 \%$ inoculum) at the moment of inoculation.

\subsection{Hydrogel synthesis}

Graphene hydrogels (GHs) were produced by self-assembly of GO through chemical reduction of the aqueous GO sheets over different conductive mesh-works: plain carbon cloth (CC) or a 304 stainless steel mesh (SS, mesh 50). The GO flakes were prepared according to the Tour's method. ${ }^{27} \mathrm{~A} 9: 1 \mathrm{H}_{2} \mathrm{SO}_{4} / \mathrm{H}_{3} \mathrm{PO}_{4}$ mixture was prepared. In an Erlenmeyer flask $1.0 \mathrm{~g}$ of graphite flakes (Sigma-Aldrich) and $6.0 \mathrm{~g} \mathrm{KMnO}_{4}$ (Cicarelli) were mixed together. Then $133 \mathrm{ml}$ of the $\mathrm{H}_{2} \mathrm{SO}_{4} / \mathrm{H}_{3} \mathrm{PO}_{4}$ mixture were poured over the solids. The reaction mixture was then heated to $50{ }^{\circ} \mathrm{C}$ with mechanical agitation for $12 \mathrm{~h}$. The reaction mixture quickly took a greenish colour and after several hours it turned brown and very thick. Then it was allowed to cool to room temperature and was poured slowly over a mixture of crushed ice $(120 \mathrm{~g})$ and $30 \% \mathrm{H}_{2} \mathrm{O}_{2}(3 \mathrm{ml})$. The obtained yellow suspension was filtered, then the filter cake was washed and filtered successively with $100 \mathrm{ml}$ distilled water, $100 \mathrm{ml} \mathrm{10 \%} \mathrm{HCl}$ (Cicarelli), 2 times $100 \mathrm{ml} \mathrm{EtOH}$ (Cicarelli) and finally the solid was coagulated with $\mathrm{EtO}_{2}$ (Cicarelli), filtered, allowed to dry in the hood overnight and placed in the oven at $45{ }^{\circ} \mathrm{C}$ for 1 hour. The GO suspension was prepared at concentrations of $6.0 \mathrm{mg}$ $\mathrm{ml}^{-1}$ in deionised sterile water with $1 \mathrm{~h}$ of ultrasonication. The biggest aggregates (a very little amount) were separated by 3 cycles of $60 \mathrm{~min}$ centrifugation at $4000 \mathrm{rpm}$, with collection of the supernatant and the solid residues trashed away.

\subsection{Preparation of electrodes}

The GH electrodes were prepared as follow: a $0.6 \mathrm{~cm}$ diameter, $8 \mathrm{~cm}$ long glass tube was tapped on the bottom side with a rubber cap. A $6 \mathrm{~cm}$-long mesh (a $0.6 \mathrm{~cm}$-wide band in the case of CC or a $0.6 \mathrm{~cm}$-wide tube in the case of SS) was introduced in the tube, then $6 \mathrm{ml}$ of an ascorbic acid (AA) and GO solution (AA-GO solution, prepared by dissolving 5 mass equivalent of ascorbic acid in aqueous $\mathrm{GO}$ at $6 \mathrm{mg} \mathrm{ml}^{-1}$ ) was poured into the tube (approximately $5 \mathrm{~cm}$ of the mesh was submerged in the solution). Then the top of the tube was sealed with a rubber cap and placed in oven at $40{ }^{\circ} \mathrm{C}$ for $60 \mathrm{~h}$. During this time, the brownish GO colloid turned to a black rGO hydrogel. The rubber cap was removed and the glass tube containing the gel was placed in a beaker with excess distilled water for washing out over $72 \mathrm{~h}$, replacing the water 3 times every $24 \mathrm{~h}$. Lastly the 
electrode was pushed away from the tube and the upper part of the mesh not submerged into the GO was electrically connected by a crocodile clip to the electrochemical circuit. The resulting materials were used as anodes in MECs. For comparison purposes, pristine CC or SS meshes of comparable size without $\mathrm{GH}$, and a solid graphite rod (constructed from bars of $0.4 \mathrm{~cm}$ in diameter and $10 \mathrm{~cm}$ in length), were also used as anodes in control experiments. The graphite rods were polished with sandpaper (P1000 grit), thoroughly rinsed with distilled water and sonicated in a bath (1 minute, 3 times). Additional control experiments in the absence of bacteria were performed. Three independent experiments were performed for each electrode type.

\subsection{MEC design and operation}

The MECs consisted of sterile anaerobic glass single-chamber cells $(200 \mathrm{ml})$ with three electrodes, where the prepared electrodes served as working electrode, a coiled platinum wire was used as counter electrode and a $\mathrm{Ag} / \mathrm{AgCl}$ electrode was used as reference electrode. The cells were filled with culture media, flushed continuously with $\mathrm{N}_{2}: \mathrm{CO}_{2}(80: 20)$ and operated in an incubator at constant temperature $\left(30^{\circ} \mathrm{C}\right)$ and stirring. The working electrode was poised at $+240 \mathrm{mV}$ vs. $\mathrm{Ag} / \mathrm{AgCl}$ (PGSTAT101 potentiostat, Metrohm AG) and the MECs were inoculated with $10 \%$ stationary-phase cultures of G. sulfurreducens. Sodium acetate $(30 \mathrm{mM})$ was provided as the electron donor, and no electron acceptor other than the electrode was present in the MEC. The MECs were changed from batch to continuous mode $24 \mathrm{~h}$ after inoculation, with a hydraulic retention time of $24 \mathrm{~h}$. The current intensity was recorded for 10 days.

\subsection{Electrochemical measurements}

Cyclic voltammetry (CV) was performed scanning the potential between $+240 \mathrm{mV}$ and $-550 \mathrm{mV}$ starting from $240 \mathrm{mV}$ (PGSTAT101 potentiostat, Metrohm AG). The scan rate was $1 \mathrm{mV} \mathrm{s}^{-1}$. CVs were performed without stirring and without gas flushing. CVs were run before each inoculation. CVs were also performed over mature biofilms before the end of the experiment, in turnover (in the presence of acetate) and non-turnover conditions (in the absence of acetate). Thus, for non-turnover conditions G. sulfurreducens mature biofilms were starved (the electrolyte was replaced with a medium that lacked acetate) until catalytic features were significantly reduced (less than $10 \%$ of maximum current density remained).

\subsection{Scanning electron microscopy}

Electrodes were fixed in glutaraldehyde $2.5 \%$ over-night, dehydrated by immersion in a series of ethanol solutions $(40,60,80$, and $100 \%$ ethanol in ultra-pure water), critical point dried, and sputtered with gold for the observation by SEM. Samples were observed in a FEI-NOVA Nano SEM at an accelerating voltage of $5 \mathrm{kV}$.

\subsection{Raman spectroscopy}

Raman spectra of samples of GH (excluding the supporting meshes) were acquired with a Lab Ram HR Evolution, Jobin Yvon, Horiba Scientific Raman Spectrometer, using a $633 \mathrm{~nm}$ Laser, 250 hole, 600 grooves per $\mathrm{mm}$ grid. $100 \times$ objective, and a $800 \mathrm{~cm}^{-1}$ to $3200 \mathrm{~cm}^{-1}$ spectral range, except stated otherwise. The laser power at the source was set to 2 watts (or $30 \mathrm{~A}$ current) and internal filter were used to attenuate the out coming power. Except stated otherwise, usual spectra acquisition conditions were: 4 accumulations of $60 \mathrm{~s}$ each, at $1 \%$ transmitted laser power, with other setting as previously stated. Other studied conditions were $1 \%, 5 \%, 10 \%$ laser power; 2 or 4 accumulations.

\subsection{Fourier transformed infrared spectroscopy}

Samples of $\mathrm{GH}$ were collected after critical point dried and grinded with $\mathrm{KBr}$, and then compressed into thin pellets. The FTIR spectra were obtained on a Fourier Transform Infrared Spectrometer (Spectrum 400-Perkin-Elmer).

\section{Results and discussion}

\subsection{Performance of GH anodes in MEC}

The performance of the electrodes was analysed in terms of the material stability and current generation during MEC operation.

The mechanical stability of the bio-electrodes is very important for its long-term operation. Manipulation and stirring may cause irreversible damage or detachment of substantial portions of the electrode, shortening its durability. Comparing our two models, GH-SS and GH-CC, the former showed a higher durability since the core of the hydrogel is protected wrapped behind the mesh. On the other hand, since in GH-CC the hydrogel is laid on the carbon cloth, it was more prone to detachment during normal operation. For this reason, although our two electrodes showed similar performances in terms of current generation, GH-SS was preferred for long-term MEC operation.

No current generation was appreciated after $240 \mathrm{~h}$ of operation of the MECs operated with the pristine meshes, CC and SS, in the presence of bacteria, as well as in any of the MECs operated in the absence of bacteria (data not shown). Therefore, the performance of the $\mathrm{GH}$ electrode, in terms of current production, was compared to that of a graphite rod (gr), one of the most employed electrode materials in BES.

The results of current generation, in the presence of bacteria, are shown in Fig. 1 as the anodic (oxidation) current density, considering the geometric area of the portion of the electrodes submerged in the electrolyte. This calculation does not take into account neither the porosity of the materials nor the electroactive surface area. However, considering that the oxidation reaction is carried out inside the microorganisms, these parameters do not necessarily represent proper markers of available surface for catalysis. For example, since the size of a typical bacterium is $0.5-3.0 \mu \mathrm{m}$, increasing the specific surface 


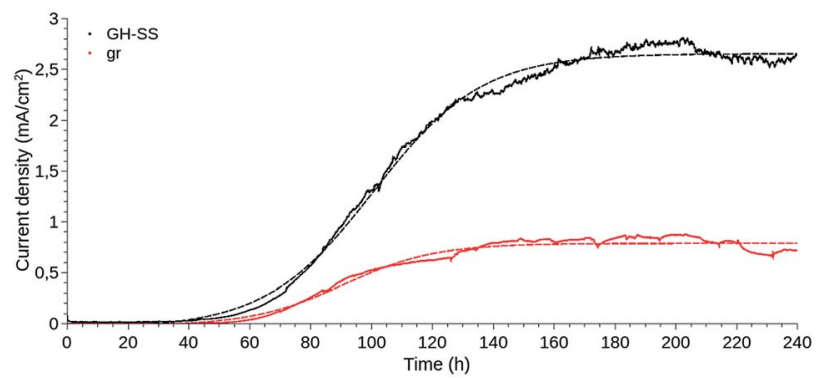

Fig. 1 Amperometric response of graphite rod (gr) and graphene hydrogel-based (GH-SS) working electrodes as a function of time, during $G$. sulfurreducens biofilm growth, under $+240 \mathrm{mV}$ vs. $\mathrm{Ag} / \mathrm{AgCl}$ applied potential. The plotted data are the mean of three independent experiments performed for each electrode type (-). The Boltzmann fit was performed with QtiPlot software and is shown for each electrode (- - -). Current density was calculated considering the geometric area of the portion of the electrodes submerged in the electrolyte.

area of the electrode does not imply a proportional increase in the surface area available for bacterial colonisation.

GH-SS displayed a better performance than gr, reaching a current density approximately three times higher.

The current density evolved likewise for GH-SS and gr, in a manner resembling bacterial growth curves: a lag phase for cell attachment and initial colonisation, followed by a fast exponential growth phase, which indicated that the electrogenic bacteria were transferring electrons to the anode as a result of intra-cellular acetate oxidation, and a stationary phase where the current stabilised and thus the biofilms were considered mature. The lag phase of GH-SS as well as gr lasted $\approx 40 \mathrm{~h}$, during this period cells go through an adaptation process from using a soluble (fumarate) to a solid electron acceptor and begin the biofilm development by secreting exo-polymeric substances. The exponential phase was longer for the GH-SS than the gr, suggesting that $\mathrm{GH}$ is capable of sustaining the growth of a higher number of bacterial cells. Accordingly, $1.5 v s .0 .8 \mathrm{mg}$ of protein per $\mathrm{cm}^{2}$ were extracted in alkali for GH-SS and gr respectively (determined by Bradford method ${ }^{29}$ ).

To further understand the impact of the material on the biofilm behaviour, we fitted two different models to the amperometries, shown in Fig. 1.

First, a logistic model (eqn (1)) was applied on the assumption that the lag, exponential and plateau phases observed for the curves of current density $v s$. time parallel similar phases of the biofilm growth. ${ }^{30,31}$

$$
q(t)=\frac{\operatorname{Max}}{1+\exp \left(\frac{4 \mu(\lambda-t)}{\operatorname{Max}}+2\right)}
$$

Thus, $\mu$ (the tangent in the inflection point) reflects the bacterial maximum growth rate; $\lambda$ (the $x$-axis intercept of this tangent) is the lag time and Max (the asymptote) represents the carrying capacity, the maximum population size that the environment can sustain. The $\mu$ and Max values are significantly higher for GH-SS $\left(\mu=3.8 \times 10^{-2} \mathrm{~mA} \mathrm{~cm}^{-2} \mathrm{~h}^{-1}\right.$ and $\mathrm{Max}=2.7$
$\left.\mathrm{mA} \mathrm{cm}{ }^{-2}\right)$ than gr $\left(\mu=1.3 \times 10^{-2} \mathrm{~mA} \mathrm{~cm}^{-2} \mathrm{~h}^{-1}\right.$ and $\mathrm{Max}=0.8$ $\mathrm{mA} \mathrm{cm}{ }^{-2}$ ), while $\lambda$ are very similar $(62.3 \mathrm{~h}$ and $59.5 \mathrm{~h}$ for GH-SS and gr, respectively). Considering that all the electrochemical cells were operated at the same conditions (inoculum size, temperature, culture media, HRT), a possible explanation of these results is a promoting effect of GH-SS on the biofilm metabolism that affects the growth or substrate oxidation rate. As discussed later, Fig. 5 shows that in our electrodes GH exposes a considerable amount of hydrophilic groups which could enhance its wettability ${ }^{26}$ and proton transport, and thus improve bacteria-electrode connection and metabolic rates.

An alternative explanation is that GH-SS exposes a larger active surface capable of being colonised by the microorganisms. It is worth mentioning that the effective electrode area depends on the geometry of pores and bacteria, the number of actual contacts per microorganism, which in turn may be affected by chemical characteristics of the material. Thus, the current physical methods employed to estimate solvent exposed areas are of little avail in our case. Therefore, as stated before, the geometric area of the electrode was employed to calculate the current density in the amperometries. But, as shown later by SEM images Fig. 4, bacteria are able to reach intimately the porous structure of $\mathrm{GH}$, consequently the actual active surface could be much greater for GH-SS and the current density overestimated.

To address these hypothesis, we also fitted a Boltzmann equation (eqn (2)) to the amperometries. ${ }^{32}$

$$
q(t)=\frac{\operatorname{Max}}{1+\exp \left(\frac{t_{50}-t}{\theta}\right)}
$$

In this equation, Max is the highest current achieved and the slope $(\theta)$ at the inflection point $\left(t_{50}\right)$ is independent of the number of bacteria, and reflects an intrinsic growth rate. Applying this model, similar slopes were expected if the only difference between $\mathrm{GH}$ and gr were the amount of active surface and the biofilms were growing at the same rate. From the $\theta$ values obtained (17.7 and $15.0 \mathrm{~mA} \mathrm{~cm}{ }^{-2} \mathrm{~h}^{-1}$ for $\mathrm{GH}$ and gr, respectively), GH shows a higher slope suggesting that both, physicochemical and active surface contributions, could explain its increased bacterial loading capacity and better performance.

Finally, we estimated the rate of acetate consumption and supply under continuous operation in our MECs. Considering the following equation for acetate metabolic oxidation, eqn (3),

$$
\begin{aligned}
1 / 8 \mathrm{CH}_{3} \mathrm{COO}^{-}+3 / 8 \mathrm{H}_{2} \mathrm{O} \rightarrow & 1 / 8 \mathrm{CO}_{2}{ }^{+} \\
& 1 / 8 \mathrm{HCO}_{3}^{-}+\mathrm{H}^{+}+\mathrm{e}^{-}
\end{aligned}
$$

bacteria produce 1 Coulomb per $1.29 \mu \mathrm{mol}$ of acetate. Therefore, the rates of acetate consumption, calculated at the highest current production are $46.4 v s .29 .0 \mu \mathrm{mol} \mathrm{h}^{-1}$ for GH and gr, respectively. Since the inflow of acetate is $2.5 \mathrm{mmol} \mathrm{h}^{-1}$, we conclude that our MECs operate at excess of acetate even if the efficiency of acetate to electrons conversion is bellow $100 \%$. 
Interestingly, considering the electrodes bacterial loading previously mentioned (1.5 vs. $0.8 \mathrm{mg}$ of protein per $\mathrm{cm}^{2}$ for $\mathrm{GH}^{-}$ SS and gr, respectively), we calculated that the acetate consumption rate per $\mathrm{mg}$ of protein is 1.6 times higher for $\mathrm{GH}^{-}$ SS than gr ( $7.8 v s .4 .7 \mu \mathrm{mol} \mathrm{h}^{-1}$ per $\mathrm{mg}$ of protein, respectively). An equal acetate consumption rate per $\mathrm{mg}$ of protein would have been expected if the only difference between GH-SS and gr was the increased active surface, which allowed a higher bacterial loading capacity. Instead, this finding supports the hypothesis that the GH chemical properties favours the acetate oxidation reaction.

\subsection{Macro-porous structure of GH anodes and electrogenic biofilm establishment}

The presence of G. sulfurreducens biofilms in GH anodes was confirmed at day 4 of MEC operation as a characteristic red coating over the electrode surface (Fig. 2).

SEM images of the electrodes surface at the end of the experiments showed that dense biofilms grew over GH displaying heterogeneous pillar structures, similarly to graphite rod (gr) control electrodes (Fig. 3A and B). Only a few bacteria were adhered to the SS and CC pristine meshes (Fig. 3C and D), explaining the absence of a significant current production for these electrodes.

Cross-sectional SEM images of GH without bacteria (Fig. 4A and B) revealed a hierarchical pore structure, with a broad size distribution of interconnected porous, typical of freeze-dried graphene hydrogels. ${ }^{33}$ This structure is the result of the partial overlapping of the flexible graphene sheets during the hydrogel formation. Cross-sectional SEM images of GH taken at the end of the experiments revealed a thick layer of cells $(>50 \mu \mathrm{m})$ on the graphene facing the electrolyte (Fig. 4C). As observed in Fig. 4D, graphene sheets seem to be intercalated between the firsts layers of cells of the biofilm. Bacteria were found along the entire GH framework (Fig. 4E). However, the core of the electrode was not clogged with microorganisms, probably because the pore sizes of the carbon material range in the order of bacteria dimensions, which might prevent a denser bacterial growth. This situation might improve substrate diffusion and ion transport within the electrode and therefore bacterial catalysis, as well as bacteria-electrode direct interaction and charge transfer. Pili-like filaments were present along the thick biofilm (Fig. 4F). Pili are required for optimal biofilm

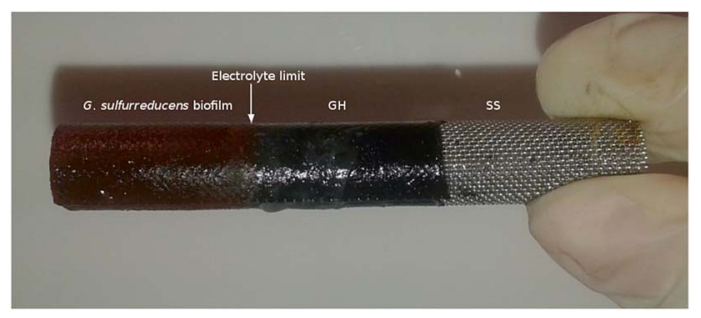

Fig. 2 Graphene hydrogel-based working electrode (GH-SS, stainless steel) at the end of the MEC operation. The red coating of the electrode is the $G$. sulfurreducens mature biofilm.

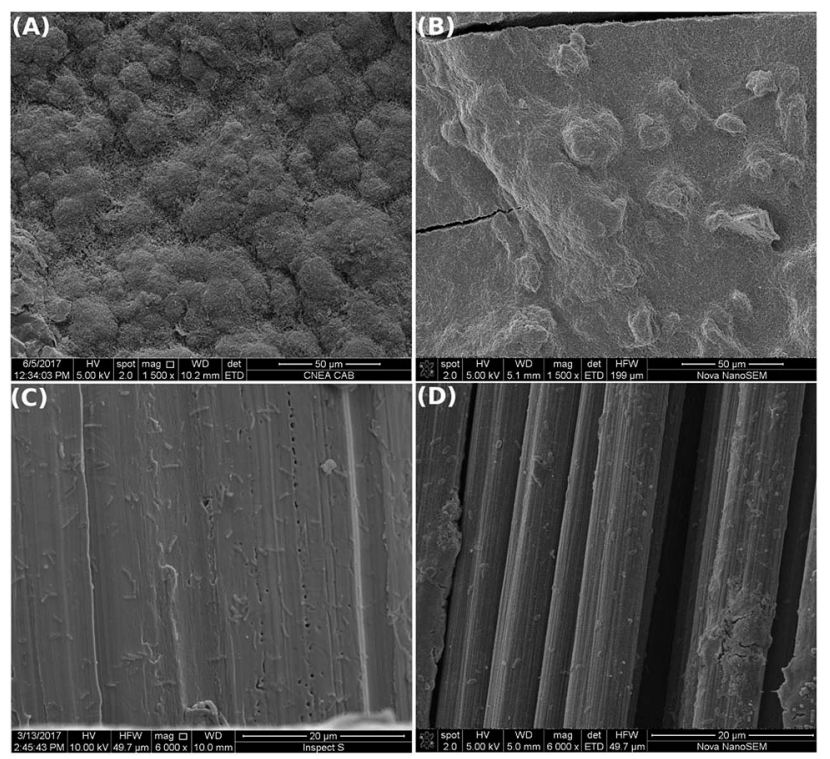

Fig. 3 SEM images of the electrodes surfaces after MEC operation in the presence of a pure culture of $G$. sulfurreducens: in (A) GH-SS and (B) gr: a dense biofilm was observed over the electrode surface; while in (C) SS and (D) CC only a few cells were found adhered to the electrodes surface.

development and for the establishment of an electrical connection with extra-cellular electron acceptors, promoting long-range electrical transfer. ${ }^{34-36}$

Therefore, GH has an enhanced active area per electrode volume capable of increasing the bacterial loading capacity. GH electrodes were able to sustain the development of dense and thick electrogenic biofilms over its surface and inside its framework, favouring an enhanced current production as observed in Fig. 1.

\subsection{Chemical characteristics of $\mathrm{GH}$ anodes and bacterial interaction}

Despite the chemical reduction step, a common feature of rGO is the presence of oxygen-containing functional groups, such as hydroxy, carboxy or epoxy groups. ${ }^{37}$ Some of these oxygencontaining functional groups are present in $\mathrm{GH}$, as determined by FTIR and Raman spectroscopy (Fig. 5). The FTIR spectrum of GH before MEC inoculation presented an absorption band at $3200-3600 \mathrm{~cm}^{-1}$, characteristic of $-\mathrm{OH}$ groups; an absorption band at $\approx 1690-1760 \mathrm{~cm}^{-1}$, attributed to the $\mathrm{C}=\mathrm{O}$ bond; and $\mathrm{C}-\mathrm{O}$ vibrations at $\approx 900-1200 \mathrm{~cm}^{-1}$. Finally, the skeletal vibration of $\mathrm{C}=\mathrm{C}$ is observed at $\approx 1620-1680 \mathrm{~cm}^{-1}$. In Raman spectroscopy, the $\mathrm{G}$ band (at $\approx 1582 \mathrm{~cm}^{-1}$ ) is associated with bond stretching of the $\mathrm{sp}^{2}$ carbon pairs, while the $\mathrm{D}$ band (at $\approx 1350 \mathrm{~cm}^{-1}$, absent in high quality graphene) is observed in all $\mathrm{sp}^{2}$ disordered carbon materials, and thus high $\mathrm{D} / \mathrm{G}$ ratios indicate higher ordered in the material. ${ }^{38}$ Interestingly, in our experiments, GH was further reduced during the MEC operation as observed in the FTIR (Fig. 5A) as well as Raman spectra (Fig. $5 \mathrm{~B}, \mathrm{D} / \mathrm{G}=1.70$ vs. 2.05 before and after operating the MEC respectively). This reduction of $\mathrm{GH}$ can be attributed to bacterial 

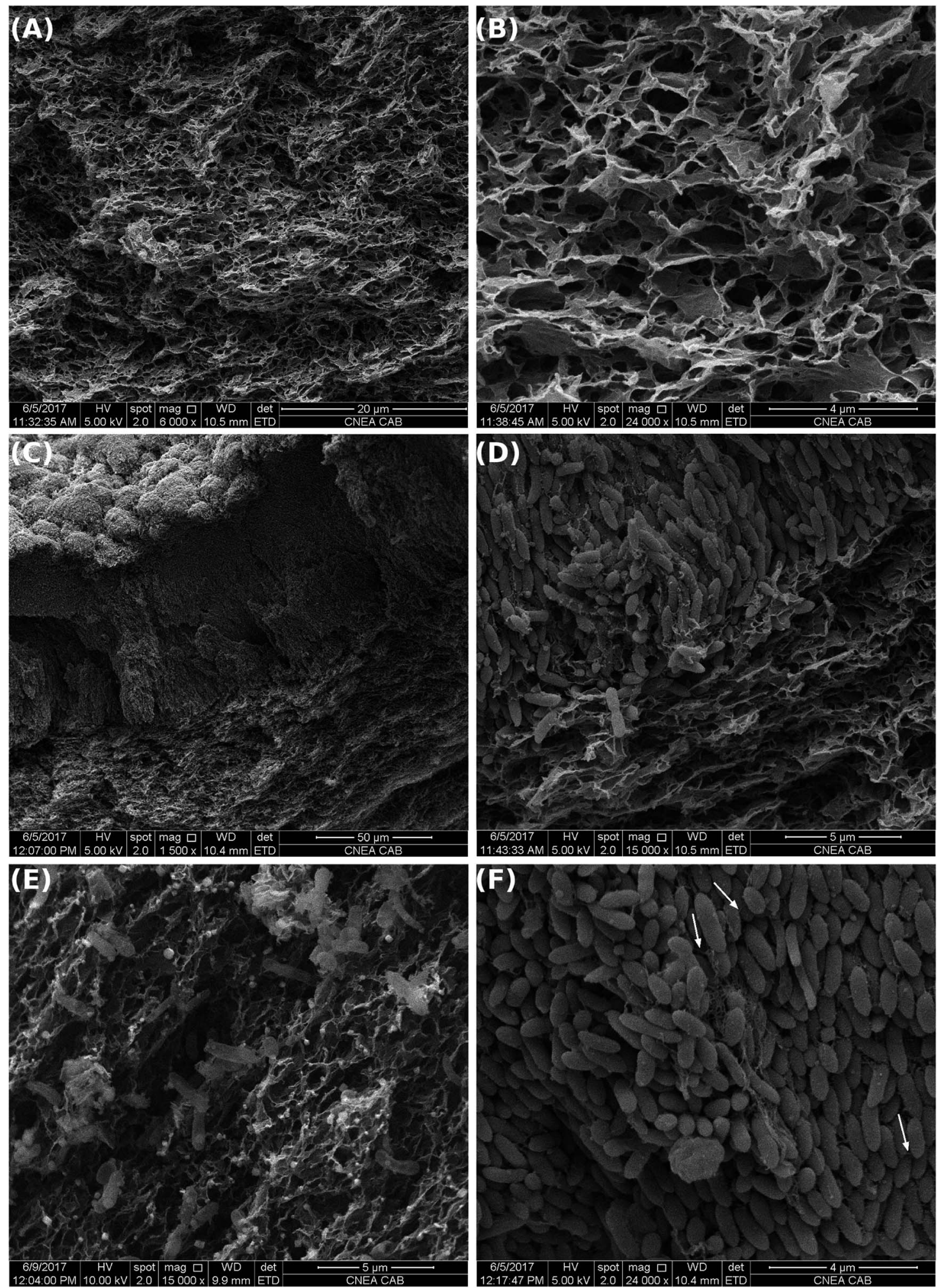

Fig. 4 Cross-sectional SEM images of graphene hydrogel-based electrode before ( $A$ and $B$ ) and after (C-F) MEC operation in the presence of a pure culture of $G$. sulfurreducens. (A) Graphene hydrogel cross-sections at (A) low and (B) high magnifications, where the macro-porous structure and pore sizes of the material can be appreciated. (C) Graphene face in contact with the bulk electrolyte after MEC operation, where a thick layer of cells can be observed. (D-F) Higher magnification of (C): in (D) graphene sheets can be distinguished between bacterial cells; in (E) bacterial cells were present in the entire GH framework; in (F) pili-like filaments were observed along the thick biofilm (some of them are pointed by arrows). 


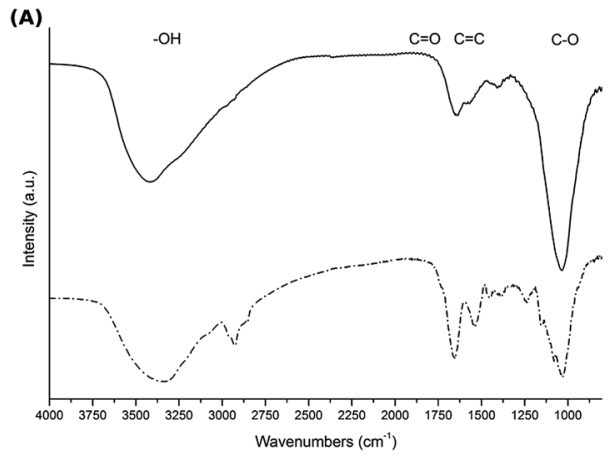

(B)

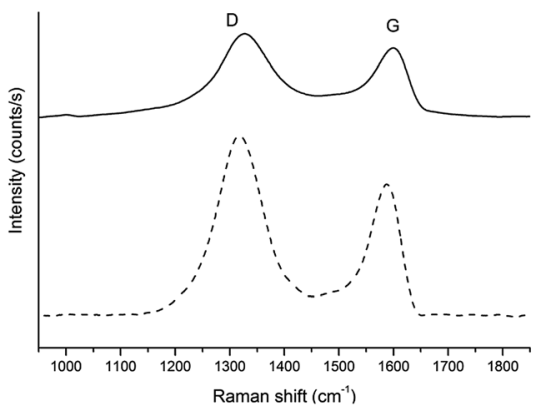

Fig. 5 (A) FTIR and (B) Raman spectroscopy of GH before (-) and after (- - -) MEC operation in the presence of a pure culture of $G$. sulfurreducens.

activity since the irreversible electrochemical reduction of GO occurs at $-1039 \mathrm{mV}$ vs. $\mathrm{Ag} / \mathrm{AgCl} .^{39,40}$ Additionally, after MEC operation, a band at around $2900 \mathrm{~cm}^{-1}$ appeared in the FTIR spectrum, consistent with the presence of alkyl groups abundant in cellular membranes. ${ }^{41,42}$

The presence of hydrophilic functional groups in GH may favour water adsorption onto the graphene sheets, preventing them from re-stacking and stabilising a porous structure with higher surface area. In addition, this feature, considered a defect in graphene, might be beneficial for bacterial-electrode interaction. ${ }^{43}$ On the one hand, the formation of a biofilm is affected by multiple factors such as the surface topography, charge and wettability, the amount of exo-polymer production by the cells, and cell-cell signalling. It was suggested that wettability enhances the connection between the bacteria and the electrode by altering local polarity at their interface, and extreme hydrophobicity makes high quality graphene unfavourable for bacterial adhesion. ${ }^{26}$ On the other hand, it was reported that the maximum current densities produced by electrogenic biofilms are limited by respiration inhibition due to proton accumulation inside the biofilm during the substrate oxidation. ${ }^{44}$ It was demonstrated that in GO, hydrophilic sites help to proton diffusion through hydrogen-bonding networks along the adsorbed water film, turning GO into a highly protonconductive material. ${ }^{45}$ Therefore, an enhanced proton conduction in GH containing some degree of oxygenated groups, might enable a faster substrate oxidation, resulting in a faster current production compared to gr, explaining the differences in the slopes observed in the exponential phase of the amperometries.
(A)
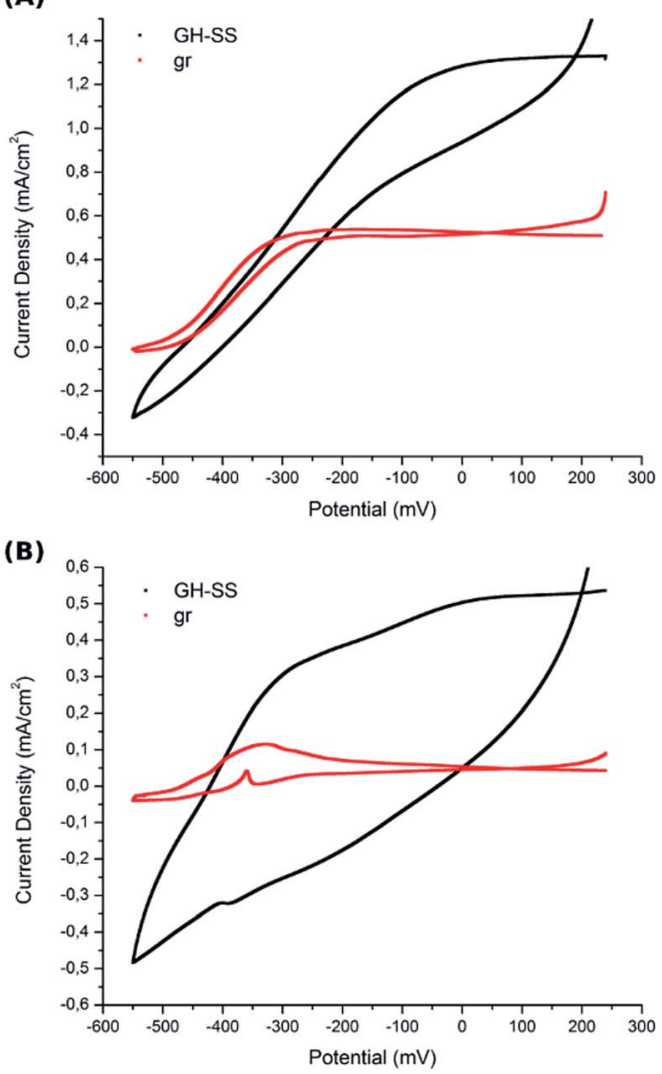

(C)

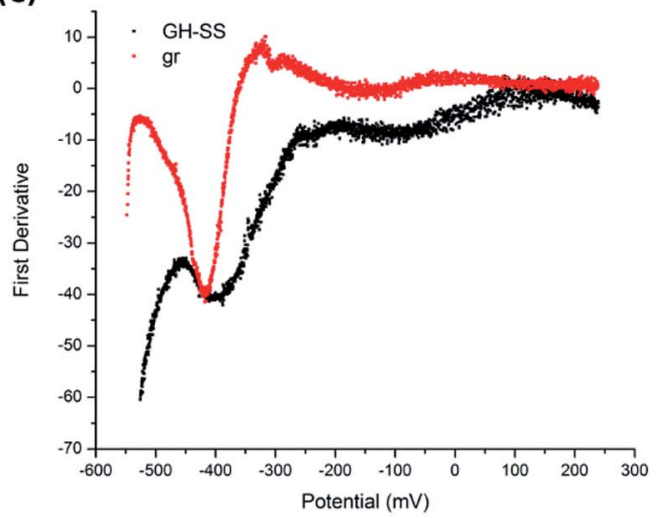

Fig. 6 Cyclic voltammetry of graphite rod (gr) and graphene hydrogel-based (GH-SS) working electrodes recorded at a scan rate of $1 \mathrm{mV}$ $\mathrm{s}^{-1}$ in (A) turnover conditions (in the presence of acetate) and (B) nonturnover conditions (in the absence of acetate). (C) First derivative analysis of anodic scan from non-turnover conditions voltammogram (obtained with the potentiostat controlling software, Nova-Metrohm). CVs were performed at $240 \mathrm{~h}$ of MEC operation over mature biofilms, where the maximum current density has been reached. The plotted data are the mean of three independent experiments performed for each electrode type.

\subsection{Biofilm electron transfer process in $\mathbf{G H}$ anodes}

Electron transfer from the electrogenic microorganisms towards the anode is coupled to energy conservation, where the electrode functions as a solid electron acceptor during respiration. A great variety of electron transport chains are found 
among microorganisms. ${ }^{\mathbf{4 6 - 4 8}}$ It was observed that changes in environmental conditions can trigger the expression of a different EET in a bacteria. ${ }^{49}$ Most studies suggest that $G$. sulfurreducens biofilms grow on carbon-based anodes by means of direct electron transfer employing two combined strategies: the secretion of non-diffusing mediators (cytochromes) into the biofilm matrix and the production of conductive pili or nanowires that enable the connection between the cells and the electrode. ${ }^{3}$

In order to gain insight into bacterial EET to the electrode surface on GH-SS anodes, cyclic voltammetry (CV) was carried out under different conditions. First, $\mathrm{CV}$ was performed at a low scan rate $\left(1 \mathrm{mV} \mathrm{s}^{-1}\right)$ in the presence of acetate, so that the enzymes that catalyse slow reactions can undergo multiple turnovers at each sampled potential. The voltammograms of both GH-SS and gr electrodes (Fig. 6A), indicate that G. sulfurreducens mature biofilms behave as attached bio-catalysts with heterogeneous electron transfer to the electrode, as previously reported. ${ }^{50,51}$ Also, both voltammograms show the anodic scan crossing under the cathodic scan at potentials higher than $0 \mathrm{mV}$ vs. Ag/AgCl. This phenomenon has already been observed for full-grown $G$. sulfurreducens biofilms on a graphite cloth anode. ${ }^{52}$ The catalytic process started in both electrodes at $\approx-490 \mathrm{mV}$. However, it was limited at $-325 \mathrm{mV}$ in gr and at $-100 \mathrm{mV}$ in GH-SS. This effect may be related to the hierarchical pore architecture of the $\mathrm{GH}$ that allows a better substrate diffusion inside the framework.

Under turnover conditions the high catalytic current recorded by $\mathrm{CV}$ may obscure individual redox species that could account for EET identification. Thus, CV was also performed in non-turnover conditions (in the absence of acetate): the G. sulfurreducens mature biofilms were starved (the electrolyte was replaced with a medium that lacked acetate) until catalytic features were significantly reduced (less than $10 \%$ of maximum current density remained); then CV was performed at a low scan rate $\left(1 \mathrm{mV} \mathrm{s}^{-1}\right)$. Fig. 6B shows voltamogramms of $\mathrm{GH}-\mathrm{SS}$ and gr, which display peaks associated with more than one redox couple. Examination of the first derivative of anodic scan from Fig. 6B voltammogram, reveals that the potential of the oxidation peaks is similar in both electrode materials (Fig. 6C) and is consistent with cytochromes redox potentials. ${ }^{53}$ This result suggests that the biofilms in GH-SS and gr might employ the same EET to transfer electrons to the electrode surface. We are currently performing more experiments to confirm this.

\section{Conclusions}

Graphene hydrogel-based electrodes (GH) have been successfully prepared by self-assembly of graphene oxide through chemical reduction over conductive meshes. The GH proved to be suitable to develop dense and conductive biofilms of Geobacter sulfurreducens, and produces an outstanding current density compared to commonly used gr as well as other reported graphene-based electrodes. ${ }^{54}$ This result is attributed to the large surface area and pore sizes, provided by the 3D macroporous structure of the $\mathrm{GH}$, as well as the material chemical properties, that renders a high bacterial loading capacity, and an enhanced mass transport and proton conduction, avoiding thus saturation in the bacteria-mediated acetate oxidation reaction. In conclusion, by coupling the electrode morphology with composition modulation, the GH-SS design presented here upgrades both the long-term stability and output performance of MEC. Noteworthy, this performance was obtained in a cell operated at continuous mode, which resembles a real wastewater treatment coupled to energy production application. Considering that the graphene production method employed in this work allows the preparation of large quantities of graphene and that the anode bio-process studied is shared by many of the BES applications, the presented results turn GH into a promising material for the development of BES technology.

\section{Conflicts of interest}

There are no conflicts to declare.

\section{Acknowledgements}

This work was supported by Comision Nacional de Energia Atomica (CNEA) and Consejo Nacional de Investigaciones Cientificas y Tecnicas (CONICET), Argentina. Partial support by Fundacion YPF (project: PIO CONICET-YPF 13320130100203CO). Authors acknowledge Tec. P. Troyon and Tec. M. Corte for SEM images acquisition; Lic. P. Sessin for Raman spectra acquisition and processing; Dr L. Fernandez Albanesi for FTIR acquisition-Centro Atomico Bariloche-CNEA; and Dr J. P. Busalmen for his advices and kindly providing $G$. sulfurreducens cultures, INTEMA-Univ. Nac. Mar del Plata, Argentina.

\section{Notes and references}

1 D. R. Bond, D. E. Holmes, L. M. Tender and D. R. Lovley, Science, 2002, 295, 483-485.

2 D. R. Lovley, Nat. Rev. Microbiol., 2006, 4, 497-508.

3 F. Kracke, I. Vassilev and J. O. Kromer, Front. Microbiol., 2015, 6, 1-18.

4 D. R. Lovley, Annu. Rev. Microbiol., 2012, 66, 391-409.

5 D. R. Bond and D. R. Lovley, Appl. Environ. Microbiol., 2003, 69, 1548-1555.

6 B. E. Logan, B. Hamelers, R. Rozendal, U. Schröder, J. Keller, S. Freguia, P. Aelterman, W. Verstraete and K. Rabaey, Environ. Sci. Technol., 2006, 40(17), 5181-5192.

7 B. E. Logan, D. Call, S. Cheng, H. V. M. Hamelers, T. H. J. A. Sleutels, A. W. Jeremiasse and R. A. Rozendal, Environ. Sci. Technol., 2008, 42, 8630-8640.

8 J. B. a. Arends and W. Verstraete, Microb. Biotechnol., 2012, 5, 333-346.

9 A. Escapa, R. Mateos, E. J. Martínez and J. Blanes, Renewable Sustainable Energy Rev., 2016, 55, 942-956.

10 B. E. Logan and K. Rabaey, Science, 2012, 337, 686-690.

11 P. Liang, K. Xiao, Y. Zhou, X. Zhang and B. E. Logan, Environ. Sci. Technol, 2009, 43, 7148-7152. 
12 K. P. Nevin, S. A. Hensley, A. E. Franks, Z. M. Summers, J. Ou, T. L. Woodard, O. L. Snoeyenbos-west and D. R. Lovley, Appl. Environ. Microbiol., 2011, 77, 2882-2886.

13 H. Wang, H. Luo, P. H. Fallgren, S. Jin and Z. J. Ren, Biotechnol. Adv., 2015, 33, 317-334.

14 Y. Zhao, Z. Li, J. Ma, H. Yun, M. Qi, X. Ma, H. Wang, A. Wang and B. Liang, J. Hazard. Mater., 2018, 349, 168-176.

15 X. Jiang, J. Shen, K. Xu, D. Chen, Y. Mu, X. Sun, W. Han, J. Li and L. Wang, Water Research, 2018, 130, 291-299.

16 F. Sun, H. Liu, B. Liang, R. Song, Q. Yan and A. Wang, Bioresour. Technol., 2013, 143, 699-702.

17 Y. Liu, Y. Zhang, X. Quan, J. Zhang, H. Zhao and S. Chen, Bioresour. Technol., 2011, 102, 2578-2584.

18 C. I. Torres, A. K. Marcus, H. S. Lee, P. Parameswaran, R. Krajmalnik-Brown and B. E. Rittmann, FEMS Microbiol. Rev., 2010, 34, 3-17.

19 H. Yuan and Z. He, Nanoscale, 2015, 7, 7022-7029.

20 K. S. Kim, Y. Zhao, H. Jang, S. Y. Lee, J. M. Kim, K. S. Kim, J.-h. Ahn, P. Kim, J.-y. Choi and B. H. Hong, Nature, 2008, 457, 706-710.

21 C. Lee, X. Wei, J. W. Kysar and J. Hone, Science, 2008, 321, 385-389.

22 K. S. Novoselov, V. I. Fal, L. Colombo, P. R. Gellert, M. G. Schwab and K. Kim, Nature, 2012, 490, 192-200.

23 Y. Zhang, G. Mo, X. Li, W. Zhang, J. Zhang, J. Ye, X. Huang and C. Yu, J. Power Sources, 2011, 196, 5402-5407.

24 J. Liu, Y. Qiao, C. X. Guo, S. Lim, H. Song and C. M. Li, Bioresour. Technol., 2012, 114, 275-280.

25 X. Cao, Z. Yin and H. Zhang, Energy Environ. Sci., 2014, 7, 1850-1865.

26 Y.-C. Yong, X.-C. Dong, M. B. Chan-Park, H. Song and P. Chen, ACS Nano, 2012, 6, 2394-2400.

27 D. C. Marcano, D. V. Kosynkin, J. M. Berlin, A. Sinitskii, Z. Sun, A. Slesarev, L. B. Alemany, W. Lu and J. M. Tour, ACS Nano, 2010, 4, 4806-4814.

28 R. Atlas, Handbook of Microbiological Media, 4th edn, 2010, 978-1-4398-0408-7, Ebook-PDF.

29 M. Bradford, Anal. Biochem., 1976, 72, 248-254.

30 M. H. Zwietering, I. l. Jongenburger, F. M. Rombouts and K. van't Riet, Appl. Environ. Microbiol., 1990, 56, 1875-1881.

31 D. Verotta, J. Haagensen, A. M. Spormann and K. Yang, Comput. Math. Methods Med., 2017, 2017.

32 J. M. Dubois, G. Ouanounou and B. Rouzaire-Dubois, Prog. Biophys. Mol. Biol., 2009, 99, 87-93.

33 X. Zhang, Z. Sui, B. Xu, S. Yue, Y. Luo, W. Zhan and B. Liu, J. Mater. Chem., 2011, 21, 6494-6497.

34 D. L. Cologgi, S. Lampa-pastirk, A. M. Speers, S. D. Kelly and G. Reguera, Proc. Natl. Acad. Sci., 2011, 108, 15248-15252.
35 G. Reguera, K. P. Nevin, J. S. Nicoll, S. F. Covalla, T. L. Woodard and D. R. Lovley, Appl. Environ. Microbiol., 2006, 72, 7345-7348.

36 G. Reguera, R. B. Pollina, J. S. Nicoll and D. R. Lovley, J. Bacteriol., 2007, 189, 2125-2127.

37 D. R. Dreyer, S. Park, C. W. Bielawski and R. S. Ruoff, Chem. Soc. Rev., 2010, 39, 228-240.

38 A. C. Ferrari and D. M. Basko, Nat. Nanotechnol., 2013, 8, 235-246.

39 L. Chen, Y. Tang, K. Wang, C. Liu and S. Luo, Electrochem. Commun., 2011, 13, 133-137.

40 Y. C. Yong, Y. Y. Yu, X. Zhang and H. Song, Angew. Chem., Int. Ed., 2014, 53, 4480-4483.

41 V. Ragoonanan, J. Malsam, D. R. Bond and A. Aksan, Biochim. Biophys. Acta, Biomembr., 2008, 1778, 2283-2290.

42 H. Muhamadali, Y. Xu, D. I. Ellis, J. W. Allwood, N. J. Rattray, E. Correa, H. Alrabiah, J. R. Lloyd and R. Goodacre, Appl. Environ. Microbiol., 2015, 81, 3288-3298.

43 J. A. Cornejo, C. Lopez, S. Babanova, C. Santoro, K. Artyushkova, L. Ista, A. J. Schuler and P. Atanassov, J. Electrochem. Soc., 2015, 162, 597-603.

44 C. I. Torres, A. K. Marcus and B. E. Rittmann, Biotechnol. Bioeng., 2008, 100, 872-881.

45 M. R. Karim, K. Hatakeyama, T. Matsui, H. Takehira, T. Taniguchi, M. Koinuma, Y. Matsumoto, T. Akutagawa, T. Nakamura, S.-i. Noro, T. Yamada, H. Kitagawa and S. Hayami, J. Am. Chem. Soc., 2013, 135, 8097-8100.

46 M. E. Hernandez and D. K. Newman, Cell. Mol. Life Sci., 2001, 58, 1562-1571.

47 G. Reguera, K. D. Mccarthy, T. Mehta, J. S. Nicoll, M. T. Tuominen and D. R. Lovley, Nature, 2005, 435, 10981102.

48 J. A. Gralnick and D. K. Newman, Mol. Microbiol., 2007, 65, 111.

49 E. Marsili, D. B. Baron, I. D. Shikhare, D. Coursolle, J. a. Gralnick and D. R. Bond, Proc. Natl. Acad. Sci. U. S. A., 2008, 105, 3968-3973.

50 E. Marsili, J. B. Rollefson, D. B. Baron, R. M. Hozalski and D. R. Bond, Appl. Environ. Microbiol., 2008, 74, 7329-7337.

51 K. P. Katuri, P. Kavanagh, S. Rengaraj and D. Leech, Chem. Commun., 2010, 46, 4758-4760.

52 H. Richter, K. P. Nevin, H. Jia, D. a. Lowy, D. R. Lovley and L. M. Tender, Energy Environ. Sci., 2009, 2, 506.

53 P. S. Jana, K. Katuri, P. Kavanagh, A. Kumar and D. Leech, Phys. Chem. Chem. Phys., 2014, 16, 9039-9046.

54 Y.-Y. Yu, D.-D. Zhai, R.-W. Si, J.-Z. Sun, X. L. Yong and Y.-C. Yong, Int. J. Mol. Sci., 2017, 18, 90. 